\title{
MolE - A Communication Service Between Modelica Compilers and Text Editors
}

\author{
Nicola Justus ${ }^{1} \quad$ Christopher Schölzel $^{1} \quad$ Andreas Dominik $^{1} \quad$ Thomas Letschert $^{1}$ \\ ${ }^{1}$ KITE, Technische Hochschule Mittelhessen, Giessen, Germany, \{nicola. justus, christopher.schoelzel, \\ andreas.dominik, thomas.letschert\}@mni.thm.de
}

\begin{abstract}
The Modelica language is becoming increasingly popular among scientists and engineers as platform for modelling physical or biological systems. Although Modelica is maintained as non-proprietary language by the Modelica Association, a considerable number of commercial implementations and development environments is complemented by a surprisingly small number of open source tools.
\end{abstract}

In this paper, we present the communication service MolE that connects any text editor as front-end with a Modelica compiler as back-end. Based on the simple HTTP communication protocol, editor plugins for a software developer's favourite text editor can be developed easily, hence turning any editor into a lightweight Modelica development tool.

We also present a first implementation of a plugin for the text editor Atom that exhibits features necessary for efficient software development, such as display of compile errors, code completion, go to declaration or view of context-sensitive documentation. In addition, Modelicaspecific checking of the number of equations in a model is supported.

Keywords: Modelica, open source, integrated development environment, distributed systems, structured editor, ENSIME, OpenModelica, JModelica, MoTE

\section{Introduction}

Modelica is a powerful object-oriented programming language that facilitates acausal description of physical systems. Although many commercial and open source tools for developing or working with Modelica are available, the OpenModelica suite (Fritzson et al., 2005) is the only comprehensive set of tools for Modelica. OpenModelica provides a standalone Modelica compiler, an Eclipse plugin for developing Modelica inside of Eclipse (MDT), a graphical model editor for connecting components (OMEdit), and a Modelica debugger. The primary tools for developing Modelica are MDT and OMEdit. Both are full-fledged integrated development environments (IDEs).

IDEs are well suited for working with big projects but may have some disadvantages. They often are slow, difficult to use and and may be even scary for novice users. For Modelica additional challenges arises from the differences between Modelica compilers, such as JModelica or OpenModelica which silghtly differ in their understanding of Modelica. In order to develop code compatible with different compilers, the IDE should be able to compile models using different compilers.

Today, when writing source code or any other type of structured text, it is common to use a structured editor which is aware of the document's structure. Structured editors are an essential part of most IDEs. Experienced developers usually prefer them to other - graphical - means of input. A structure aware editor must be able to analyze the text given to it. Thus structure awareness means awareness of the syntax and to some extend also of the semantics of the texts it deals with. The structured editor is deeply integrated with the IDE, rather than being just a mere component.

In this paper we present Modelica I Editor (MolE), a development environment for Modelica, centered on editing and checking complex models, refraining form all issues of model execution. A structured editor is its main component and user interface.

A key concept of MolE is that the user may use a text editor of her own choice, attach it to a service process that provides syntactic and semantic analysis and transforms the plain text editor to a structured editor.

Thus users may edit texts using the editor they are used to and still benefit from automatic recompilation, code completion, semantic highlighting, go to declaration, refactoring, and so on.

A central part of our solution is a server process that mediates between the text editor and Modelica aware analytical services. These services are provided by existing Modelica compilers, and/or further existing or future tools that may be plugged into this infrastructure (Figure 1). We have enhanced one text editor to a Modelica editor, but other text editors may be integrated with little effort. These editors only have to provide a plugin that implements the service API. This API provides a unique interface to different Modelica compilers and eases the communication with compilers and related tools, protecting users from complex and differing command-line interfaces.

The design of MolE was inspired by the ENSIME project (ENSIME Contrib., 2016) with its server process that mediates between text editors and Scala compilers.

MolE is an environment for developing Modelica models 
using editors that are enhanced to be Modelica aware. It was realized as part of the first author's bachelor's thesis (Justus, 2016).

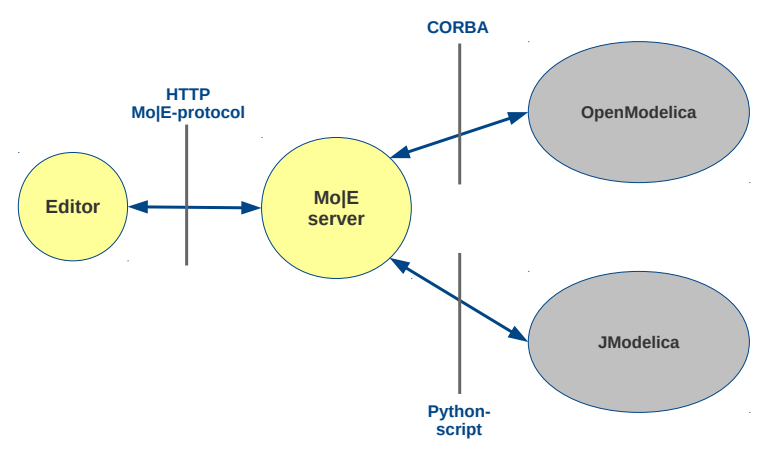

Figure 1. Survey of the communication between a text editor with a MolE-plugin, the MolE server and OpenModelica or JModelica.

\subsection{Structure of the Paper}

Section 2 describes which technologies and standards where used to implement MolE. Section 3 describes the protocol between client and service process, the communication with OpenModelica as well as with JModelica. Section 4 presents the key features of Modelica I Editor (MolE) and their use in the text editor Atom. Finally, section 5 gives a summary and a short outlook on future extensions.

\subsection{Naming}

The name Modelica I Editor (MolE) alludes to the use of the pipe character (I) in UNIX-like operating systems, which establishes a pipeline between two programs. MolE can be seen as such a pipeline between the Modelica compiler and a structured editor. In contexts where special characters like the pipe may cause problems, we chose the alternative spelling Modelica-Pipe-Editor (MoPE).

\subsection{Goals}

Our goals for MolE are:

- Provide an extendable client server application which makes it possible to develop Modelica inside existing text editors.

- Provide a client implementation for the text editor Atom as reference for other clients.

- Highlight syntax and type errors, perhaps while typing, inside the text editor.

- Provide code completion for models, data types, and variables.
- Provide jump to the source of a model. This is better known as "go to declaration".

- Provide a view of the documentation of a model.

\subsection{Background and Related Work}

OneModelica (Samlaus, 2015) is a an Eclipse-based IDE for Modelica models tailored to the domain of fluid dynamics. It was realized using tools and techniques of Model Driven Software Development. It may be compared to our approach in that it restricts itself to syntax and static semantics of the language and refrains from simulation issues. It differs considerably in its technological base, which in the years since its development has lost a lot of its attraction and support, not without reason as we think.

MolE is the first tool in a more ambitious project called Modelica Tool Ensemble (MoTE). MoTE aims at the provision of a collection of small user-friendly standalone applications for developing and executing Modelica models, i.e. a lightweight development environment for Modelica.

Modelica does not differ in principle from other languages when it comes to development environments. However, due to its complex static and dynamic semantics, it poses special challenges, mainly for the support of incremental development (see e.g. (Höger, Lorenzen, and Pepper, 2010) or (Broman, Fritzson, and Furic, 2006)).

We are well aware of these problems. Thus, at least for the time being, MoTE and MolE do not include a Modelica compiler or tools incorporating compiler features much beyond parsing. Instead we rely on mature compilers like OpenModelica and JModelica.

\section{Technologies}

\subsection{Scala and Akka}

Scala (EPFL, 2016) is a hybrid programming language that combines object orientation with functional programming. Because the Scala compiler generates bytecode for the Java Virtual Machine (JVM), it integrates with many available Java libraries. In addition, resulting compiled programs are platform independent. The service process of MolE is implemented in Scala.

Akka is a library for concurrent and distributed systems, based on the actor model that facilitates concurrency by providing a high level of abstraction (Allen, 2013). We use Akka as a provider of communication services, such as an implementation of the HTTP-protocol and for structuring the system according the actor model.

\subsection{OMC and CORBA}

OpenModelica provides the Advanced Interactive OpenModelica Compiler (OMC), a server that provides an API to query loaded Modelica code (Asghar et al., 2011).

The Common Object Request Broker Architecture (CORBA) is used by the OpenModelica compiler server $\mathrm{OMC}$ as interface to other applications and other programming languages. 
CORBA developed by the Object Management Group (OMG) defines a standard for inter-process communication modeled as interaction of distributed objects. Because the public API of remote objects is defined in an Interface Definition Language (IDL), processes may be implemented in different programming languages (OMG, 2012).

\subsection{Atom and the Electron Engine}

Atom (GitHub, 2016a) is a text editor created by GitHub in the style of Sublime Text (Sublime HQ Pty Ltd, 2016). Basic design concepts of Atom include customization and extensibility through plugins (called packages in the context of Atom). Extending Atom is possible with JavaScript, HTML and CSS by using the Electron Engine (GitHub, 2016c). This allows to rapidly develop extensions and to implement communication protocols using AJAX requests. Furthermore, Atom already includes a package for syntax highlighting for Modelica (Chenouard et al., 2016), a simple API for completion suggestions (GitHub, 2016b) and a plugin for clicking on text (Facebook, 2016), which is used to implement go to declaration functionality.

We have created an Atom plugin as first reference implementation of a MolE client.

\section{Design}

\subsection{MolE - Editor Protocol}

Clients are connected to the service process, by means of Hypertext Transfer Protocol (HTTP)-based communication and JavaScript Object Notation (JSON) data representation. HTTP provides status codes, Uniform Resource Identifiers (URIs) and content negotiation (Fielding and Reschke, 2014). JSON is a compact text format, based on the JavaScript Object Notation (Bray, 2014).

The communication flow follows several steps: Firstly, the client connects to the service process using a connect request that communicates the current project. In this context a project is a directory containing Modelica source files.

Secondly, after initialization the service process answers with the respective project id. The unique project id identifies the project in the client server communication.

Henceforth, the client uses this project id to request further IDE functionality for this project from the service process.

To finally finish a session, the client sends a disconnect request that triggers the service process to delete all projectrelated information and cached data.

The following sections describe each supported IDE functionality in more detail.

\subsubsection{Connecting to the server}

As introduced in the preceding section, each client needs to connect initially with the server. A connect request is initiated through a POST request containing the respective JSON object with the project description. The JSON object contains the full path into the project directory and the relative path to a directory that is used to store compiled files:

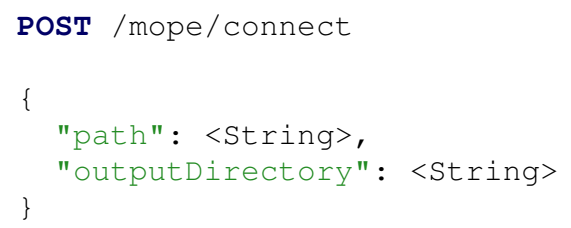

This project information is stored in the mopeproject. json file that is placed in the project directory.

If the request was successful, the server answers with a project id. If not, the server answers with 400 BadRequest and a detailed error message.

\subsubsection{Compiling Modelica source files \& Modelica script files}

Compiling a Modelica source file is initiated through a compile request. The request body contains the path to the currently opened file. As a result of the request a model is instantiated and type errors are retrieved:

PosT /mope/project/0/compile

\{ "path": <String> \}

If the request was successful, the server answers with a JSON array containing compiler errors:

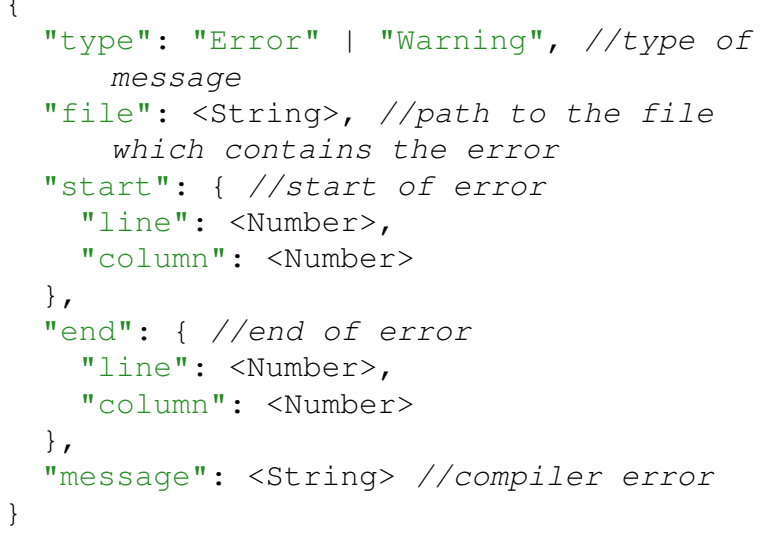

Compiling a Modelica script file is initiated by sending an analogous compilescript request:

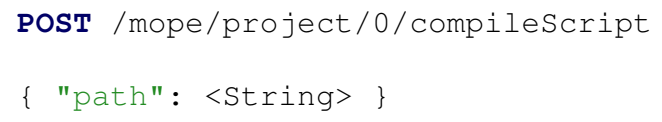

Although the request is called "compiling a Script file", the service process actually executes the script. This action is intended for debugging purposes of smaller scripts and not for scripts that simulate a model, since simulating a model is time-consuming and may freeze or possibly even kill the service process.

\subsubsection{Checking a model}

To check a model for its number of equations the client sends a checkModel request with the model path. The server calls the OpenModelica compiler to run checkModel and answers with a string containing the results: 
POST /mope/project/0/checkModel

$\{$ "path": <String $>$ \}

This functionality is only available, if the OpenModelica compiler is used.

\subsubsection{Go to declaration}

To retrieve the declaration of a model, the client sends a declaration request. This request contains the model/class name as query string ${ }^{1}$ :

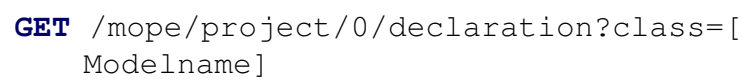

The server answers with a JSON object containing the file path and line number of the declaration:

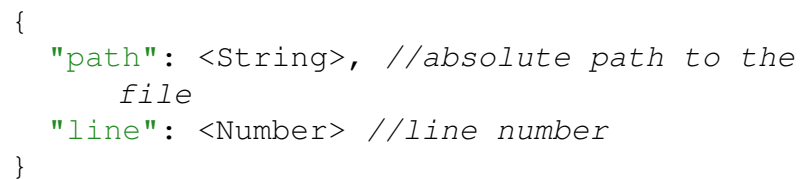

If the project id is unknown or the query string is missing, the server will answer with a 404 NotFound error.

\subsubsection{Go to documentation}

A model documentation can be retrieved using a doc request with the model name encoded as query string:

\section{GET/mope/project/0/doc?class=[Modelname]}

The server embeds the documentation in a template and returns a HTML document that can be viewed in a web browser.

If the project id is unknown or the query string is missing, the server answers with a 404 NotFound error.

\subsubsection{Code completion}

For code completion the client sends a completion request with a JSON object that describes the position of the cursor as file (name of current file), line and column number (position of the cursor) and word (part of the expression to be completed):

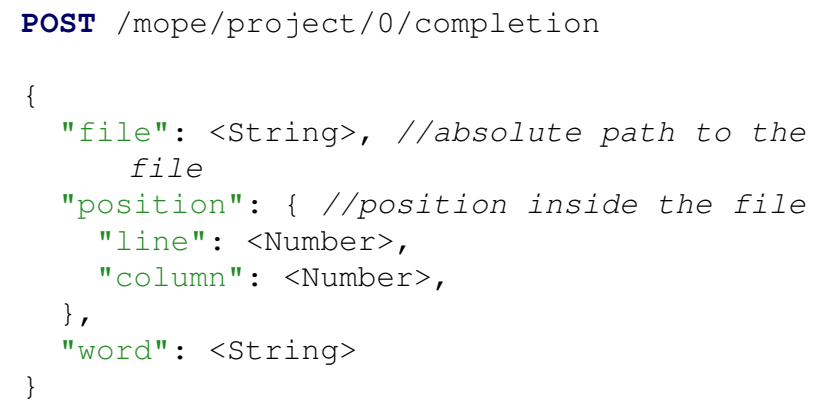

The server responds by sending an JSON array of possible completions for the expression:

\{

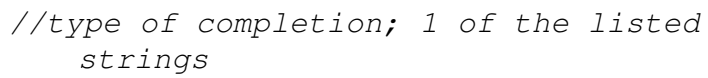

${ }^{1}$ A query string is a component of a URI, that starts with a ? (BernersLee, Fielding, and Masinter, 2005).

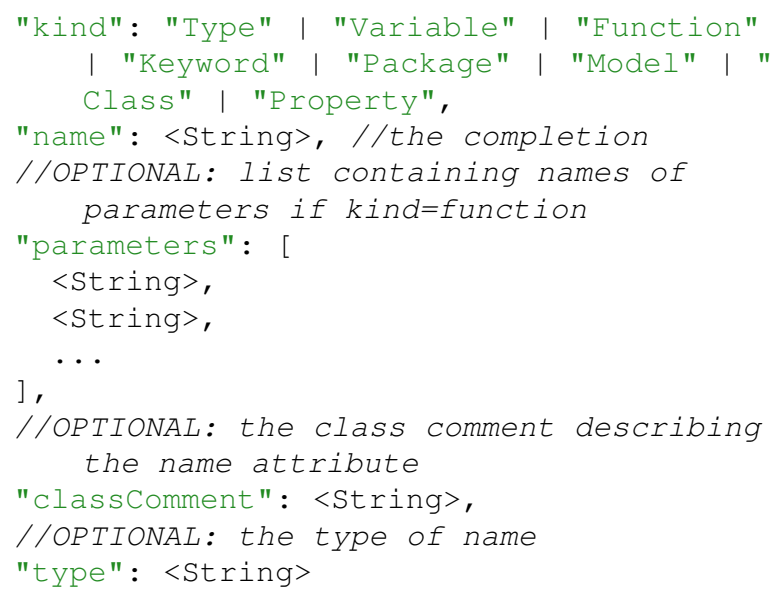

kind defines the type of the completion (such as package, class, function, variable, etc.). name is the suggestion for the subexpression.

The optional return values for parameters, classComment and type report the list of argument names if the suggestion is a function, the documentation string if the the suggestion is a class and the data type of the expression (usually the data type of a variable), respectively.

If the given project id is unknown, the server answers with 404 NotFound.

\subsubsection{Display data type of a variable}

To retrieve data type and documentation string of a variable, the client sends a typeof request with a body identical to the body of the completion request. If the request was successful, the server answers with a JSON object containing the name, type, and documentation string of the variable. Otherwise the server answers with 404 NotFound:

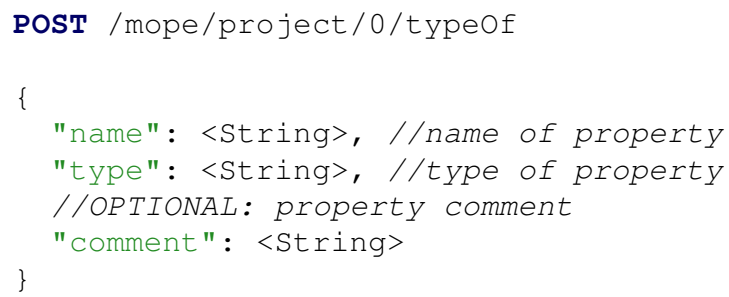

\subsubsection{Disconnecting from the server}

A session is terminated by a disconnect request, which initiates the shutdown sequence for this project on the server:

PosT/mope/project/0/disconnect

The server returns 204 NoContent if the project id is known or 404 NotFound elsewise.

\subsubsection{Stopping the server}

The client can stop the whole service process by sending a stopserver request. The server answer is $202 \mathrm{Ac}-$ cepted.

PosT /mope/stop-server 


\subsection{Communication with OpenModelica}

The modeling and development environment OpenModelica (OSMC, 2016) consists of a Modelica compiler (omc), a graphical connection editor (OMEdit), an Eclipse plugin (MDT) and a Modelica debugger (Fritzson et al., 2005). As described in Chapter 2.2 the compiler enables querying for model information via its CORBA interface that provides several types of information:

- list of all models/classes by sending getClassNames,

- source file of a model by sending get SourceFile,

- documentation annotation of a model by sending getDocumentationAnnotation,

- result of a model check for equations by sending checkModel,

- documentation string of a model by sending getClassComment,

- arguments of a function by sending getParameterNames,

- specialization of a class by sending getClassRestriction.

An additional difficulty arises from the fact that OpenModelica uses Modelica expressions as arguments for its CORBA interface. As a result, the functions listed above are not implemented explicitely in the CORBA interface. Instead, OpenModelica only provides a single method in its CORBA interface, namely sendExpress ion and sends Modelica source code strings and API function calls as arguments. Therefore, we create the function calls as strings and interpolate them into the function argument, as shown in Listing 1.

Listing 1. API function call through OpenModelica's CORBA interface.

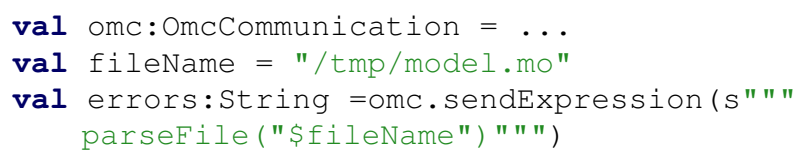

\subsection{Communication with JModelica}

JModelica (Modelon AB, 2016) is a Modelica compiler developed by Modelon AB (Åkesson et al., 2010). To allow dynamic adjustments during execution, JModelica offers a Python interface which enables code modification at run time. In addition it enables compilation of Modelica code. We are using this Python interface for compilation of the models by delivering the Modelica source files to a custom Python script, which calls the JModelica compiler, parses JModelica's output and encodes the output into JSON. The resulting JSON is printed to stdout which is afterwards parsed by the service process and finally decoded as Scala

\section{Command}

Mope: Disconnect

Mope: Compile Project

Mope: Run Script

Mope: Check Model

Mope: Show Type

Mope: Open Documentation

Mope: Open Server Log

Mope: Open Server Config

Mope: Stop Server

Table 1. List of commands implemented in the Atom plugin.

objects. The communication scheme is depicted in Figure 2.

Unfortunately JModelica does not offer access to the parsed model or its abstract syntax tree. That is the reason why code completion is restricted to local variables and go to documentation is not yet supported in the presented MolE Atom plugin.

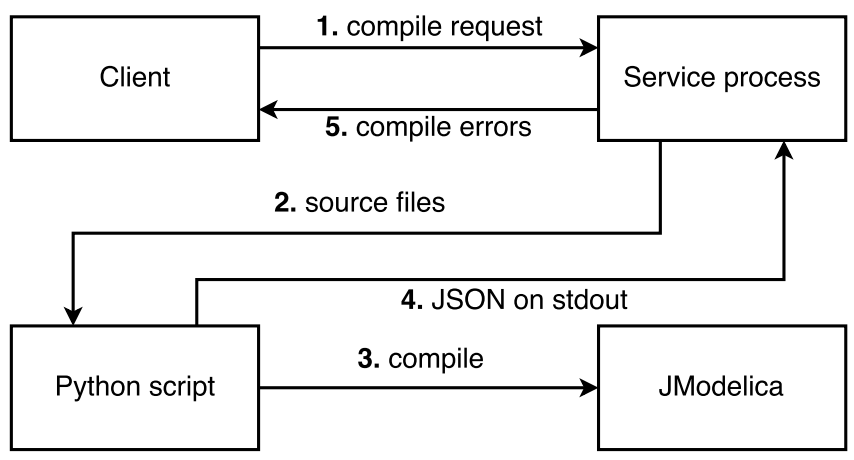

Figure 2. Diagram of the communication between a text editor (client) and JModelica.

\section{Features}

\subsection{Client commands}

Table 1 gives a full list of the commands available in the Atom plugin.

\subsection{Compiler Feedback}

Modelica I Editor (MolE) provides instant compiler feedback for syntax errors and type errors. Background compi- 
lation is automatically triggered when a file is saved and the errors are highlighted in the editor with a red indicator at the left side of the editor tab. Error messages are displayed at the bottom of the tab (Figure 3). Alternatively automatic compilation can be disabled and triggered manually.

As MolE supports JModelica and OpenModelica it is possible to use either JModelica or OpenModelica or both compilers for one project.

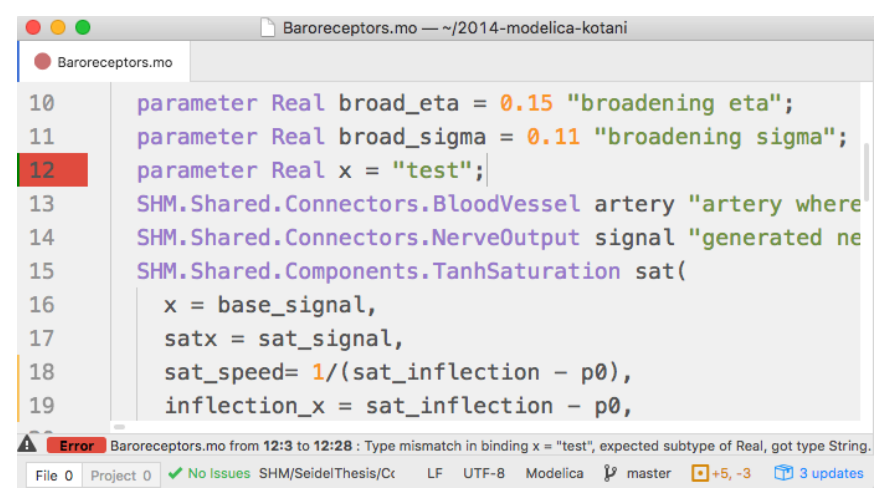

Figure 3. Compile errors are retrieved form the back-end (OpenModelica or JModelica) by the MolE server and highlighted in the source code by the editor plugin.

\subsection{Code Completion}

Modelica I Editor (MolE) features enhanced code completion on keystrokes or by pressing Ctrl + Space. Suggestions include classes, models, functions, model parameters and variables, keywords, built-in types as well as local variables. The suggestions contain a type indicator, documentation string and a link to the model's documentation (Figure 4). The type indicator displays the type of the suggestion (package, model, function or variable).

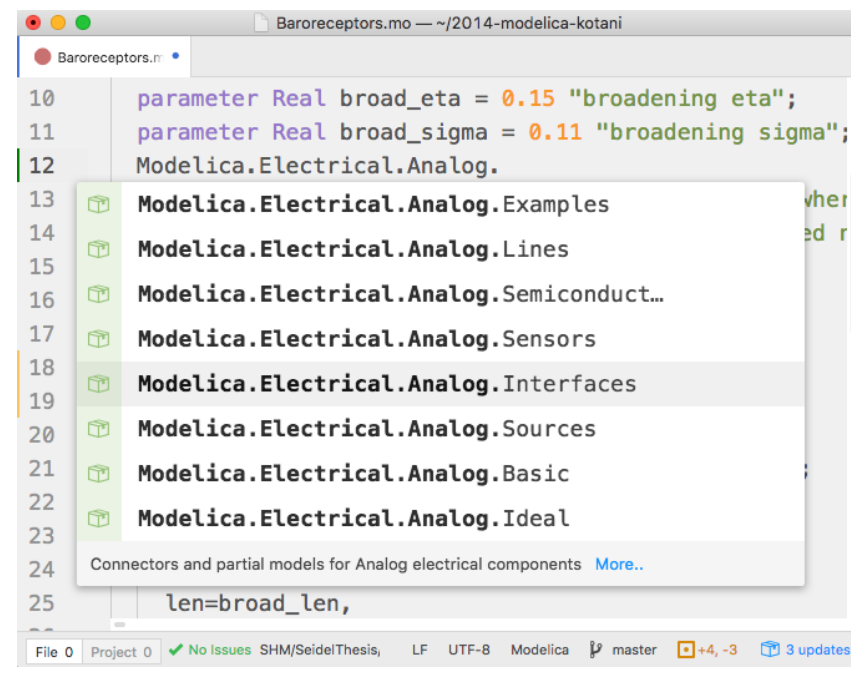

Figure 4. Code completion allows for selecting classes, models, functions, model parameters, variables, keywords or built-in types from a list of suggestions retrieved by the MolE server.

\subsection{Go to Declaration}

MolE provides go to declaration by clicking on the model/class name while holding down Ctrl. The source file of the model/class is opened in a separate tab. Go to declaration is mostly used for discovering source code or when editing multiple models that are linked to each other.

\subsection{Documentation View}

MolE embeds the queried documentation of a model in a predefined template and provides the documentation as HTML document. The implementation in the Atom plugin opens the requested documentation in the default browser. Furthermore it is possible to browse the model's child components using the links in the subcomponents section of the documentation (Figure 5).

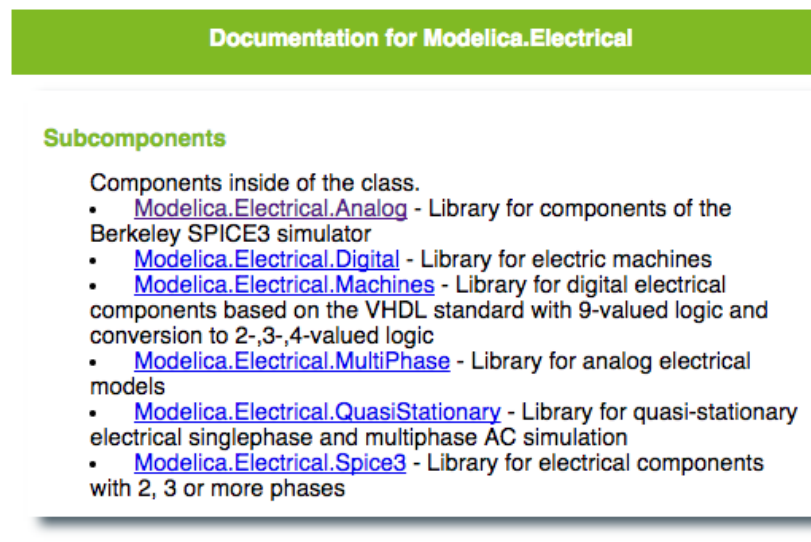

Info - Header

Info

This library contains electrical components to build up analog and digital circuits, as well as machines to model electrical motors and generators, especially three phase induction machines such as an asynchronous motor.

Documentation generated by MolE

Figure 5. Example of a documentation display generated as HTML page by MolE by embedding the retrieved documentation string with a template page.

\subsection{Type \& Documentation String Display}

MolE provides a command for displaying the type and documentation string of the variable at the cursor position. Type and documentation are displayed at the bottom of the editor tab (Figure 6). 


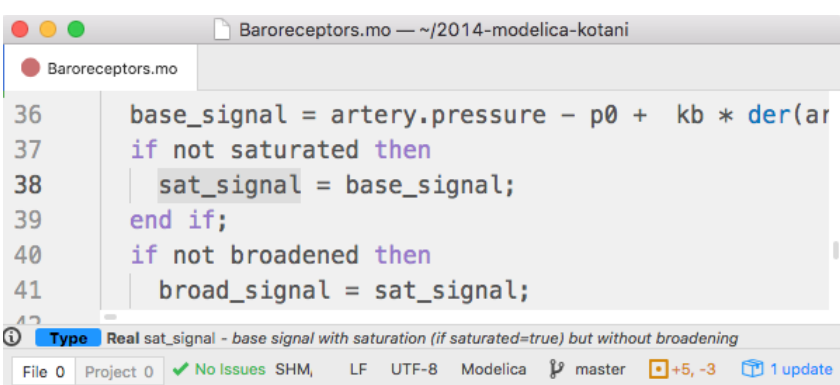

Figure 6. Data type and documentation string are displayed in the editor window by the Atom plugin.

\subsection{Execution of Modelica Scripts}

If the OpenModelica compiler is used, MolE allows manually triggered execution of Modelica scripts and displays error messages in the editor.

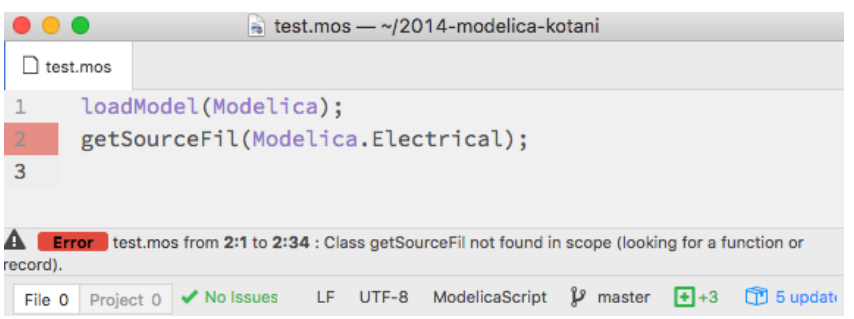

Figure 7. Compile errors of Modelica scripts are displayed in the editor window by the Atom plugin.

\subsection{Model Check}

MolE supports checking of a model for the number of equations (Figure 8), if the OpenModelica compiler is used.

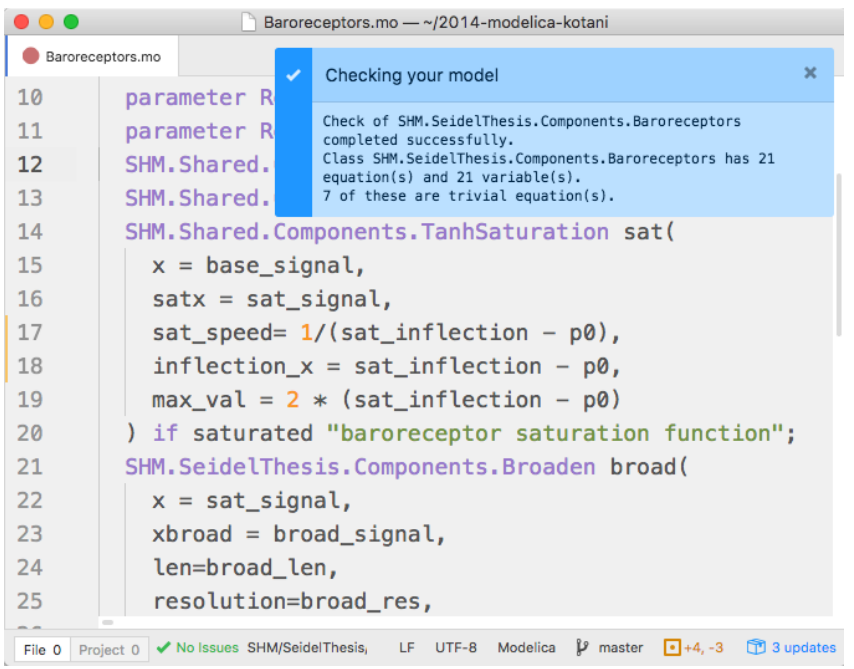

Figure 8. Result of a model check, performed by the OpenModelica back-end, is displayed as pop-up in the editor window by the Atom plugin.

\section{Conclusions}

This paper presented a extendable client/server application for developing Modelica in enhanced text editors like Atom. It shows how a service process is used to simplify communication with multiple Modelica Compilers and provide IDE features to various text editors through a simple interface. Text editors have to implement a small number of basic HTTP calls, which should be a minimal effort. A minimal setup with compilation and code completion would only require four HTTP calls. Installation instructions for MolE can be found at https: //github.com/THM-MoTE/mope-server.

$\mathrm{MolE}$ is a base for further extensions. E.g. we intend to implement plugins for different editors, such as Sublime Text (Sublime HQ Pty Ltd, 2016), Visual Studio Code (Microsoft Corporation, 2016) or vim (Moolenaar, 2016). Including Visual Studio Code should not be a problem because it uses TypeScript for its plugins, which is a superset of Atom's JavaScript.

$\mathrm{MolE}$ is part of a larger ensemble of tools called MoTE (Schölzel et al., 2016). MoTE will also include a vector graphic editor called Modelica Vector Graphics Editor (MoVE) (Justus et al., 2017) and a diagram editor called Modelica Diagram Editor (MoDE) (Hoppe et al., n.d.). Together with MolE these tools provide alternative user interfaces for the interaction with existing Modelica compilers, which allow a simpler interaction than full-fledged IDEs like OpenModelica.

The projects are open source and hosted on GitHub: https://github.com/thm-mote/

\section{References}

Åkesson, J. et al. (2010). "Modeling and Optimization with Optimica and JModelica.org - Languages and Tools for Solving Large-Scale Dynamic Optimization Problems". In: Computers \& Chemical Engineering 34 (11), pp. 1737-1749.

Allen, Jamie (2013). Effective Akka. Sebastopol, USA: O'Reilly Media.

Asghar, Syed Adeel et al. (2011). "An Open Source Modelica Graphic Editor Integrated with Electronic Notebooks and Interactive Simulation". In: Proceedings of the 8th International Modelica Conference. Dresden, Germany, pp. 739-747.

Berners-Lee, T., R. Fielding, and L. Masinter (2005). Uniform Resource Identifier (URI): Generic Syntax. RFC 3986. IETF.

Bray, T. (2014). The JavaScript Object Notation (JSON) Data Interchange Format. RFC 7159. IETF.

Broman, D., Peter Fritzson, and S. Furic (2006). "Types in the Modelica Language". In: In Proceedings of the 5th International Modelica Conference. Ed. by Ch.and Haumer A. Kral. Vienna, Austria: The Modelica Association, pp. 303-317. 
Chenouard, Raphael et al. (2016). Modelica language support in Atom. GitHub Repository. URL: https : / / github . com / modelica - tools / atom language-modelica (visited on 11/03/2016).

École Polytechnique Fédérale de Lausanne (2016). The Scala Programming Language. URL: http : / / wWw . scala-lang. org/ (visited on 11/01/2016).

ENSIME Contributors (2016). ENSIME. URL: http: / / ensime.github.io/ (visited on 11/01/2016).

Facebook (2016). Hyperclick. GitHub Repository. URL: https : / / github.com / facebooknuclide / hyperclick (visited on 11/03/2016).

Fielding, R. and J. Reschke (2014). Hypertext Transfer Protocol (HTTP/1.1): Message Syntax and Routing. RFC 7230. IETF.

Fritzson, Peter et al. (2005). "The OpenModelica Modeling, Simulation, and Development Environment". In: Proceedings of the 46th Scandinavian Conference on Simulation and Modeling (SIMS). Trondheim, Norway.

GitHub (2016a). Atom. URL: https : / / atom . io (visited on 11/01/2016).

- (2016b). Autocomplete+ Package. GitHub Repository. URL: https : / / github . com / atom / autocomplete-plus (visited on 11/03/2016).

- (2016c). Electron - Build cross platform desktop apps with JavaScript, HTML, and CSS. URL: http : / / electron.atom.io/ (visited on 09/14/2016).

Höger, Christoph, Florian Lorenzen, and Peter Pepper (2010). "Notes on the Separate Compilation of Modelica". In: 3rd International Workshop on Equation-Based Object-Oriented Modeling Languages and Tools. Ed. by P. Fritzon et al. Oslo, Norway: Linköping Electronic Conference Proceedings, pp. 43-53.

Hoppe, Marcel, Christopher Schölzel, and Andreas Dominik. MoDE - A Standalone Modelica Diagram Editor. unpublished.

Justus, Nicola (2016). "Design and Implementation of a Client/Server Application for Editing Modelica Inside Various Text Editors". BA thesis. Giessen, Germany: Technische Hochschule Mittelhessen.

Justus, Nicola, Christopher Schölzel, and Andreas Dominik (2017). "MoVE - A Standalone Modelica Vector Graphics Editor". In: 12th International Modelica Conference. Prague, Czech Republic. to be published.

Microsoft Corporation (2016). Visual Studio Code Code Editing. Redefined. URL: https : / / code . visualstudio.com/ (visited on 22/12/2016).

Modelon AB (2016). JModelica.org. URL: WWW . jmodelica.org (visited on 11/01/2016).

Moolenaar, Bram (2016). welcome home : vim online. URL: http: / / www . vim.org/ (visited on 12/21/2016).

Object Management Group (2012). Common Object Request Broker Architecture (CORBA). Part 1: CORBA Interfaces. OMG document formal/2012-11-12. Version 3.3.
Open Source Modelica Consortium (2016). OpenModelica. URL: https : / / openmodelica . org (visited on 11/01/2016).

Samlaus, Roland (2015). “An Integrated Development Environment with Enhanced Domain-Specific Interactive Model Validation". PhD thesis. Linköping University, The Institute of Technology.

Schölzel, Christopher et al. (2016). Modelica Tool Ensemble. GitHub Repository. URL: https : / / github . com/orgs / THM-MOTE/ (visited on 12/22/2016).

Sublime HQ Pty Ltd (2016). Sublime Text: The text editor you'll fall in love with. URL: https : / / wWW . sublimetext. com/ (visited on 11/01/2016). 